\title{
Innovative transformations of a model for flow distribution in gas systems and their new properties
}

\author{
Nikolay I. Ilkevich ${ }^{l}$, Tatyana.V. Dzyubina ${ }^{l, *}$, Zhanna.V. Kalinina ${ }^{l}$ \\ ${ }^{1}$ Melentiev Energy Systems Institute of Siberian branch of Russian Academy of Science (ESI SB \\ RAS), department of pipeline and hydraulic systems, Lermontov street 130, Irkutsk, Russia, 664033
}

\begin{abstract}
The methods of network analysis are widely used in mathematical modelling of optimal gas flows to solve the problems of long-term planning and development of gas systems. However, the modern development of gas systems, their renovation and modernization require that the new trends in innovative transformation should be taken into account in the mathematical models for their research as well. This paper is focused on the proposals for considering the new properties of gas system in the mathematical flow distribution model as compared to the traditional formulation. Case study involves the calculation of gas flows for an aggregated unified gas supply system for the period up to 2030. This calculation allows for the edge coefficients and a gain in the transmission capacities of the edges.
\end{abstract}

\section{Introduction}

The relevance of the research is due to the increasing scale and complexity of gas systems (GS) [1]. The desire to diversify transport corridors and to boost the maneuverability of gas flows leads to an increase in the total length and complication of the configuration of gas transmission systems. The components of the GS engineering structures are constantly improved (pipelines are made of new materials; energy-efficient compressors, high-quality shutoff and control valves, etc. are used).

Thus, the modern development of gas systems, their renovation and modernization require that the new trends in innovative transformation should be taken into account in the mathematical models for their research [2].

Melentiev Energy Systems Institute SB RAS has developed a set of mathematical models to solve complex hierarchically-subordinate problems of optimal development of future gas systems $[3,4]$.

The problems are solved at three hierarchical levels, starting from the upper level with successive detailing of solutions at lower levels of consideration. The methods of network analysis are used to solve the problems of long-term planning and development of gas systems both at the first level (a generalized problem of gas flow distribution) and at the subsequent ones, in particular, at the third level (where the reliability of a complex gas system is assessed when calculating its operating conditions).

* Corresponding author: tvleo@isem.irk.ru 
Thus, the relevance of this research is based on the need to consider the generalized problem of flow modeling in terms of an analysis and consideration of new properties of the gas system as compared to the traditional formulation.

\section{Methods of the network analysis for the study of gas system}

Due to its consistency and clarity, the network analysis based on graph theory [5] has been widely used to solve the problems of gas system operation under various conditions. These problems rest on the problem of gas transportation, i.e. the problem of the most efficient gas flow distribution in gas pipeline systems. In Russia, the researchers $[6,7,8]$, including those at the Melentiev Energy Systems Institute SB RAS [9], began to rather actively work on such problems in the late 1980s. The first software implementation was performed by V.A. Yefremov for a problem of a relatively small dimension [7].

Although the unified gas supply system of Russia knows no equals in the world, researchers in other countries also conduct the studies that examine the development of European gas system, including gas systems of different countries, and allow for gas delivery from the unified gas supply system of the Russian Federation [12, 13]. The development of gas systems for such studies is simulated using the models of gas flows. The authors of $[14,15]$ present a review and comparison of such models developed in different countries.

In the problems of flow modeling, it is convenient to consider gas system as a set of three subsystems: gas sources, main transport networks and consumers. One of the traditional statements is the problem of gas flow of the minimum cost [4].

\section{A generalized problem of flow modeling}

It is used to solve the problems of the first level [4] is written as follows:

$$
\begin{aligned}
& \sum_{(i, j}\left(c_{i j} x_{i j}+k_{i j} y_{i j}\right) \rightarrow \min \\
& \sum_{i} \lambda_{i j} x_{i j}-\sum_{i} x_{i j}= \begin{cases}-v, & j=s \\
0, & j \neq s, t \\
w, & j=t\end{cases} \\
& l_{i j} \leq x_{i j} \leq d_{i j}+y_{i j}, \quad(i, j) \in U, \\
& 0 \leq y_{i j} \leq g_{i j}, \quad(i, j) \in U .
\end{aligned}
$$

where $x_{i j}, y_{i j}$ - gas flows along the existing and new edges; $d_{i j}, g_{i j}$ - transmission capacities of the edges and their gains; $c_{i j}, k_{i j}$ - "prices" of gas transportation along the existing and new edges. $U-$ a subset of all edges. Additional nodes $s$ and $t$ are introduced, i.e. total source and sink; $v$ and $w$ - the total flows from $s$ and into $t$, respectively.

Here the optimality criterion is represented by the minimum costs of gas production, transportation and delivery to consumers (1). Constraints are represented by the production capacities of existing and new facilities and requirements to meet the minimum consumer demand (3), (4) provided there is a balance between gas supply and demand at the network nodes (2). 
Let us consider in more detail the solutions to allow for the new properties of a gas system in comparison with the traditional formulation of the flow distribution problem:

1) introduction of edge coefficient $\lambda_{i j}$ (see equation 2). This makes it possible to take into account a change in the flow $x_{i j}$ passing along the edge. It is considered that if $x_{i j}$ units of flow enter any edge $(i, j)$ at node $i$, then $\lambda_{i j} x_{i j}$ units of flow will leave this edge at node $j$. At $\lambda_{i j}>1$ this coefficient is called a payoff $[16,17]$ or edge strengthening [18]. Meanwhile at $\lambda_{i j}<1$ the flow along the edge grows weaker (is lost). In the case $\lambda_{i j}=1$, we obtain an ordinary flow problem for all sections of the network.

For the gas transmission systems, the coefficient $\lambda_{i j}$ was used to traditionally consider: auxiliary gas consumption or other gas losses (for example, due to unreliable equipment) during gas transportation or its delivery from the field or underground gas storage $\left(\lambda_{i j}<1\right)$ $[19,20]$. This coefficient, however can also be used to take into account associated small consumption (or supplies) of gas $\left(\lambda_{i j}>1\right)$ instead of introducing additional consumers, as a rule, dead-end ones (or sources), with a view to reducing the dimension of a system to be modeled [20];

2) introduction of vector of the edge transmission capacity gain $y=\left(y_{i j}\right)$ and, respectively, vector of constraints on the transmission capacity gain $g=\left(g_{i j}\right)$ in the conditions of problem (3), (4).

Consideration of these characteristics is necessary for an analysis of a model of gas system development $[4,19]$. Here the criterion of optimality is a piecewise linear convex function (see (4)). The costs proportional to the flow $\left(\sum_{(i, j)} c_{i j} x_{i j}\right)$ reflect the share of existing costs, while the costs proportional to the transmission capacity gain $\left(\sum_{(i, j)} k_{i j} y_{i j}\right)$, show the costs of the new capacity construction;

3) introduction of lower constraints $\left(l_{i j}\right)$ on flows along the edges (see (3)) [4]. This requires the construction of an additional calculation network to preliminarily estimate the possibility of the existence of a feasible solution. If the lower constraints are set correctly, i.e. the solution exists, their consideration significantly helps in the subsequent analysis of the system behavior in various specific situations. For example, when it is necessary to determine whether the existing gas system will be able to provide some consumers with the amount of gas not lower than a set level of gas consumption, or when the condition for the mandatory use of a certain share of resources at some fields or UGS is set;

4) the possibility of considering new economic conditions. In the flow model (1)-(3), the indices $c_{i j}$ are understood as specific costs or prices of self-financing related to the use of gas by the $i$-th consumer.

If to take the value of the specific profit from the use of gas at a given load node as index $c_{i j}$, the minimum cost flow problem (4) - (7) can be viewed as a profit maximization problem by rewriting the criterial function in the form

$$
\sum_{(i, j) \in \bar{U}} c_{i j} x_{i j}+\sum_{(i, j) \in U_{1}} c_{i j} x_{i j}-\sum_{(i, j) \in U_{2}} c_{i j} x_{i j} \rightarrow \min ,
$$

where, as before, $\bar{U}$ - a subset of edges $(i, j)$ of a transport network, $U_{l}$ - a subset of edges $(s, j)$, modeling gas supply sources, $U_{2}-$ a set of additional edges $(i, t)$, modeling gas consumers [4].

The problem of choosing the criterion is essential when taking into account the modern economic conditions. Various groups of criteria are proposed in [19]. None of these criteria is universal but their variations allow reflecting different interests. For example, for the flow distribution problem, at the first level, it is possible to use both the first criterion leading to savings in operating costs, and the second criterion maximizing the economic 
effect, which is very important when enterprises switch to larger economic activities. For the reliability assessment problem of a large-scale complex gas system at the third level, however, when calculating its operating conditions, the optimality criterion can be represented by the minimum of costs, given the minimum shortage of gas supply to consumers [4];

5) setting the fictitious gas prices. This makes it possible to take into account gas supply to a priority group of consumers. At the same time, the prices for each priority group should differ considerably (by an order of magnitude) from each other. By setting fictitious prices (specific indices) $c_{1}, c_{2}, c_{3}$ in (1) for each share of gas consumption so that $c_{1}<<c_{2}<<c_{3}$, it is possible to provide mandatory gas supply to the consumers of the first group under existing shortage [4].

The above-enumerated innovative transformations were successively implemented in the software "POTOK".

\section{Case study}

The database for gas systems of the Russian Federation [4] and the Energy Strategy of Russia [21] were applied to build a unified gas supply system (UGSS) design scheme and determine quasi-dynamics of its development for 2020, 2025 and 2030, given gas delivery to the countries of the Former Soviet Union and beyond [4], Table 1.

Table 1. Commissioning of new gas transportation capacities by year

\begin{tabular}{|c|c|c|c|c|c|}
\hline \multicolumn{2}{|c|}{ Tie lines } & \multicolumn{3}{c|}{$\begin{array}{c}\text { Capacity, } \\
\text { billion } \mathbf{~ m}^{3}\end{array}$} & $\begin{array}{c}\text { Share of auxiliary gas } \\
\text { consumption }\left(1-\lambda_{i j}\right)\end{array}$ \\
\hline Initial node & Final node & 2020 & 2025 & 2030 & \\
\hline Novopskov & Rostov-on-Don & 30 & & & 0,022 \\
\hline Petrozavodsk & St. Petersburg & & & 60 & 0,027 \\
\hline$\ldots$ & $\ldots$ & $\ldots$ & $\ldots$ & $\ldots$ & $\ldots$ \\
\hline Vertikos & Novosibirsk & & 60 & & 0,045 \\
\hline
\end{tabular}

The Table indicates the values of new gas transportation capacities (vectors of transmission capacity $y_{i j}$ ) and values of coefficients demonstrating reduction in the amount of auxiliary gas consumption and leakages $\left(1-\lambda_{i j}\right)$.

The calculations show that until 2030, it is necessary to introduce considerable volumes of new gas transportation and compression capacities in the main gas pipeline of the Russian Federation. This in turn activates the use of new highly maneuverable energysaving compressor-power equipment with adjustable rotation speed, pipelines from new materials, and technologies for heat insulation and waterproofing, as well as reliable and high-quality shutoff and control valves.

\section{Conclusion}

The modern development of gas systems, their renovation and modernization require that the new trends in innovative transformations be taken into account in mathematical models for their research. The following innovative transformations were proposed to solve the gas flow distribution problems while optimizing the development of gas systems. Introduce: 1) edge coefficients allowing for the change in gas flow as it passes through the edge; 2) gain vectors of the edge transmission capacities and 3) lower constraints on gas flows along the 
edges. Take into account new economic conditions: namely, 4) new criteria for optimization of flow distribution and 5) fictitious gas consumer prices which make it possible to take into account the delivery of gas to a priority group of consumers.

The proposed approaches are illustrated by the calculation of gas flows for the aggregated unified gas system for the period up to 2030. The calculation allows for the gain in the transmission capacity of edges of the gas transmission system and the coefficients showing a decrease in auxiliary gas consumption and leakage.

The research was carried out within the project III.17.4.3 of the Fundamental research program of SB RAS (AAAA-A17-117030310437-4)

\section{References}

1. N.I. Voropai, Systems Research in the Energy Sector: Retrospective Scientific Directions of SEI-ESI (2010)

2. http://www.gazprom.ru/f/posts/97/653302/prir-passport-2018-2025.pdf

3. N.I. Voropai, B.G. Saneev., et al. Energy of Russia in the XXI century (2015)

4. N.I. Ilkevich, T.V. Dzubina, Zh.V. Kalinina, Multi-level modeling of gas supply systems development (2014)

5. $\quad$ L.R. Ford, D.R. Fulkerson, Flows in networks (1962)

6. M.G. Sukharev, E.R. Stavrovsky, E.E. Bryanskikh, Optimal development of gas supply systems (1981)

7. V.A. Yefremov, Network flow models to control the operation and development of the unified gas supply system (1987)

8. M.G. Sukharev, E.R. Stavrovsky, Backing up trunk pipeline systems (1987)

9. A.P. Merenkov, E.V. Sennova, S.V. Sumarokov, et al., Mathematical modeling and optimization of heat, water, oil and gas systems (1992)

10. R. Madoliat, E. Khanmirza, A. Pourfard, Journal of Petroleum Science and Engineering, 149, 504 (2017)

11. A. Chebouba, F. Yalaoui, et al., Computers \& Operations Research, 36 (6), 1916 (2009)

12. http://www.entsog.eu/public/uploads/files/publications/TYNDP/2015/entsog_TYNDP 2015 main report lowres.pdf

13. V.A. Kulagin, T.A.Mitrova, Gas market in Europe: lost illusions and timid hopes (2015)

14. B.F. Hobbs, Imperfect Models of Imperfect Competition in EU Gas Markets, 12 (2010)

15. Y. Smeers, Gas models and three difficult (Universite Catholique de Louvain, 2008)

16. N. Christofides, Graph Theory. An Algorithmic Approach (1975)

17. N.I. Ilkevich, T.V. Dzyubina, V.V. Elnikov, S.T. Okuneva, The economy and mathematical methods, 38 (3), 25 (2002)

18. E. Minieka, Optimization algorithms for networks and graphs (1978)

19. M.G. Sukharev, Reliability of gas and oil systems (1994)

20. N.I. Ilkevich, W.I. Rabchuk, Y.D. Kononov, A Study of Methane Leakage in the Soviet Natural Gas Supply Sustem (1991)

21. Energy Strategy of Russia for the period up to 2030 (No 1715-p dated 13.11.2009) 